\title{
Human Factors of Wayfinding in Navigation
}

\author{
Daniel R. Montello ${ }^{1}$ and Corina Sas $^{2}$ \\ ${ }^{1}$ Departments of Geography and Psychology, \\ University of California, \\ Santa Barbara, California 93106, U.S.A. \\ Tel. +1 (805) 893 8536. Fax: +1 (805) 8933146. \\ montello@geog.ucsb.edu \\ ${ }^{2}$ Computing Department
Lancaster University
Lancaster LA1 4WA, UK \\ Tel. +44 (0) 15245 10318. Fax: +44 (0) 1524510492. \\ c.sas@lancaster.ac.uk
}

\section{The Psychology of Human Wayfinding}

We travel through the environment to reach places that satisfy our needs and wants. Successful travel requires that we know where to go and how to get there; it also requires that we can move along the intended route in the intended direction without having accidents or getting unnecessarily delayed. Taken together, these are requirements of navigation: coordinated and goal-directed movement through the environment. Navigation occurs over a wide spectrum of spatio-temporal scales. We navigate to the other side of the room, to the post office, to visit our relatives in another town, or to vacation half way around the world. In order to navigate effectively, we apply our psychological skills of perception, cognition, and motor behavior, within the contexts of physical and social environments, and with the assistance of technologies of information and transportation. There are consequently a host of human factors issues relevant to navigation. Attention to these issues can result in improvements to the design of technologies, environments, and training procedures that increase the ease, accuracy, efficiency, comfort, and safety of navigation. 


\subsection{Wayfinding and Locomotion: Two Components of Navigation}

Navigation may be conceptualized as consisting of the two components of wayfinding and locomotion [1]. Wayfinding refers to our requirement to know where to go and how to get there. It is the efficient goal-directed and planning part of navigation (some researchers use the term “navigation" more or less synonymously with "wayfinding”). Wayfinding requires a goal destination, a place we want to reach. This destination is typically located outside of our immediate surrounds. Thus, wayfinding is largely coordinated distally, beyond the local surrounds directly accessible to our sensory and motor systems at a given moment. Hence memory, stored internally in nervous systems and externally in artifacts such as maps, plays a critical role in wayfinding. Wayfinding requires us to solve problems involving explicit decision-making — choosing routes to take, orienting toward nonperceptible landmarks, creating shortcuts, and scheduling trips and trip sequences.

In contrast, locomotion refers to the necessarily real-time part of navigation in which we move successfully in the direction we intend without injuring ourselves or moving into obstructions. It requires coordination to the immediate surrounds directly accessible to our sensory and motor systems at a moment in time. Locomotion requires us to solve problems such as identifying surfaces of support, avoiding obstacles and barriers, and directing our movement toward perceptible landmarks. Locomotion occurs in various modes, either aided by machines (automobiles, bicycles, airplanes, and so on) or not (walking, running, and so on). There are clearly many human-factors issues relevant to locomotion, including the design of running shoes and automobile seats, the placement and appearance of dashboard gauges and building entranceways, and the banking of curves in roads. 
Most acts of navigation involve both locomotion and wayfinding components, generally as part of an integrated system that can only be separated conceptually. But you can have one without the other, as when a person nervously paces about without bumping into walls but also without "going anywhere" (only locomotion), or conversely when a person plans a trip that is never taken (only wayfinding). In this essay, we discuss human-factors issues relevant to the wayfinding component of navigation and do not consider locomotion issues further.

Successful wayfinding means that our goal destination adequately or optimally meets our needs, and that we reach it efficiently. To achieve this, we must have information about what is in the environment and where it is. As noted above, this may be stored internally in cognitive maps (belonging to the traveler or to other people who communicate it to the traveler) or externally in information displays. Effective wayfinding requires this information to be sufficiently accurate, precise, complete, and up-to-date. Furthermore, we must be able to access this information and reason with it appropriately, according to the situation we are in. It is important that the information is sufficient but not more than sufficient. For instance, excessively complete information can prevent us from focusing on relevant information by distracting us with irrelevant information. Also, the form and modality of the information is often important to the success of wayfinding.

\subsection{Orientation during Wayfinding}

A fundamental information requirement of wayfinding is that travelers are aware of their location relative to their destination, and to other places or objects (such as where important decision points are located along their route). That is, they must be oriented. Orientation with respect to locations on the earth's surface, required when people wayfind, is geographic 
orientation. But a wayfinder need not be geographically oriented completely or with great precision; in some situations, the orientation required for successful wayfinding may be quite coarse and partial. What is important is that travelers know enough to get to their destinations efficiently. It is also important that they know they have this knowledge, in part because of the negative affect that results when people are uncertain about where they are located or which way they need to go to reach a destination. This uncertainty is geographic disorientation-getting lost. Geographic disorientation may be long-lasting and very serious, even life threatening; Hill [2] presents behavioral science research relevant to the professional search and rescue that may take place in such cases. But geographic disorientation of a minor and temporary nature is very common; probably no one can truthfully say they never experience minor disorientation. Individuals with a poor sense-of-direction [3] may experience such disorientation on a regular basis. Even minor episodes of disorientation can generate anxiety, frustration, and tardiness, and during automobile travel, they contribute importantly to fuel waste, pollution, accidents, and traffic congestion.

Humans maintain orientation as they move (they update) via a combination of two processes: landmark-based and dead-reckoning processes. Landmark-based updating involves recognizing specific features or places in the world, which requires internal or external memory. Deadreckoning updating involves keeping track of components of locomotion, including velocity or acceleration (which, as vector quantities, include heading information) and travel duration (dead reckoning is sometimes called path integration). An important limitation of dead reckoning by itself is that it only provides information about the location of a new place relative to the location of another place at which one was recently located. Also, dead reckoning accumulates error if it is not periodically corrected via landmark-based processes. 


\subsection{Attention and Automaticity of Wayfinding Tasks}

Wayfinding tasks vary in their demands on attentional resources. But unlike most locomotion tasks, which are relatively automatic, most wayfinding tasks are relatively controlled or effortful. Dead reckoning over relatively short distances does not use much working-memory capacity. And wayfinding during familiar trips, such as driving between home and work, becomes automatized over time, leaving attention for other activities (though more attention is required at moments when active wayfinding decisions are being made). In contrast, maintaining orientation in unfamiliar environments over more than short distances demands attention. Considerable attentional resources are needed when giving and interpreting verbal route directions. In general, wayfinding requires controlled, explicit strategies and working-memory processes when people are in unfamiliar places, including when they are lost. These are more accurately described as reasoning processes than perceptual processes.

\section{The Environment in Wayfinding}

Navigation occurs in environments, the visual and structural characteristics of which influence the ease of wayfinding [4]. Terrestrial environments are different than aquatic and aerial environments. Relatively flat environments are different than mountainous or underground environments. These environments differ in the information they make available for wayfinding, the information about them that is required for wayfinding, and the information they provide for mapping and verbal description. An important distinction in this respect is that between built environments, created by humans, and natural environments, created relatively freely of human agency. Although this distinction is imperfect, some useful generalizations about its implications 
for wayfinding can be made. Built environments have more "regular" patterns, like straight lines and right angles. In many built environments, for example, the road network consists entirely of rectilinear grids or symmetric radial patterns. The presence of more curved, irregular, and asymmetric shapes in natural environments gives them a greater visual complexity in one sense, but at some point, this creates visual homogeneity as compared to the more minimalist character of built environments. Structures in built environments can vary greatly in terms of color and height, or they may lack variation to an extreme degree. An important difference between built and natural environments is that the first often come equipped with signs that (potentially) aid wayfinding.

Three major environmental factors have been identified that affect the ease of orientation and wayfinding [5]. The three factors are: (1) differentiation, (2) visual access, and (3) layout complexity. Differentiation is the degree to which different parts of an environment look the same or different. Environments may be differentiated with respect to size, shape, color, architectural style, and so on. Generally, more differentiated environments support wayfinding because the differentiated parts are more distinct and memorable. At some point, however, differentiation could be taken to an extreme that would be disorienting. The second factor of visual access is the degree to which different parts of an environment can be seen from various viewpoints. From which locations can travelers see their start locations, their destinations, and various landmarks along the way? Greater visual access obviously makes orientation easier. Layout complexity is a heterogeneous notion that is difficult to express in formal terms. More complex layouts typically make wayfinding more difficult. But exactly what constitutes a "complex" layout in this sense is a question for research. A more articulated space, broken up into more different parts, is generally more complex, though the way the different parts are 
organized is critical. It is clear that certain patterns of path networks are more or less complex in this sense; for example, oblique turns are more disorienting than orthogonal turns. What is difficult here, though, is that the overall shape or "gestalt" of a path layout can determine whether a particular element is disorienting. A curved street may be understood better when it fits within a radial network pattern, as long as that radial pattern is apprehended. A grid pattern may be disorienting if its axes do not run north-south and east-west—at least for those travelers who incorporate cardinal directions in their wayfinding.

Signage can be considered a fourth environmental factor. Signs (including posted maps) represent meaning symbolically in order to aid wayfinding. The design and placement of signs in the environment clearly affect orientation [6]. Unfortunately, signs can disorient too. Effective signage must be legible from a distance, must be clear and simple in design, must have enough but not too much information, and must be placed where the traveler needs information (at decision points, for instance). The challenge of designing comprehendible iconic symbols for signs is especially great; does an arrow pointing straight up mean "go forward" or "go up one floor?" With signs, as with layout complexity, many contextual factors influence effectiveness. A perfectly clear sign may be confusing if it is placed in a sea of competing visual clutter. And even the best-designed and placed signs cannot entirely make up for poor characteristics of the other three environmental factors.

\section{Information Displays for Wayfinding}

As a symbol system, signs may be considered information displays rather than environmental features. Information technologies are central to effective wayfinding. In different travel contexts and for different wayfinders, the optimal mixture of verbal and graphical information 
varies. The optimal sensory modality in which to present the information varies as well. Of primary importance is the question of visual vs. auditory information, though tactile information can be useful too, especially for travelers with visual impairments. Questions about the optimal form and modality for providing wayfinding information have recently become focused by the development of In-Vehicle Navigation Systems (IVNS), part of the broader topic of Intelligent Transportation Systems (ITS). These systems present navigational information to automobile drivers via digital displays and descriptions. They incorporate satellite Global Positioning System (GPS) technology, inertial sensors for heading, and digital geographic information systems (GIS). Their use invites a host of human-factors research questions [7]. They make traditional cartographic questions about what to display and how to avoid cognitive overload more important than ever. Furthermore, digital technologies provide a unique opportunity to tailor information displays to the individual abilities, preferences, and wayfinding styles of the traveler.

Usability studies have investigated questions about the optimal display form, modality, and layout for IVNS in different contexts (e.g., [8]). Providing map information to the driver in the visual modality has serious drawbacks if the driver attempts to read the map while steering the car. Maps are quite useful in certain driving situations, however, and preferred by some drivers. They are especially powerful for communicating complex routes. Visual displays may also present written verbal directions or symbols such as sequences of guidance arrows. The location of the visual display relative to the driver's field-of-view is important too. Head-up displays (HUD) have greater usability than low-positioned displays, since they enable drivers to access information from the display while maintaining visual contact with the road and its surroundings. Auditory displays may be especially functional as compared to maps because they free up the 
driver's visual processing for driving and provide information sequentially in a timely manner, at the moment the driver needs it. Auditory displays differ in sound quality; they can provide synthesized speech or digitized voice messages previously recorded. A general conclusion is emerging that the most functional and usable systems will display information multimodally, combining visual and auditory displays.

\subsection{Verbal Route Directions}

Verbal route directions, either written or spoken, are often the best way to provide wayfinding information. Some research investigates travelers' preference for verbal directions of different styles (e.g. [9]). Again, the advent of automated systems for wayfinding assistance motivates much of this research. Which types of features should be included in verbal directions and how should these features be described? Should the verbal directions focus exclusively on landmarks and turn instructions, or should distance be included too? Which objects in the environment should serve as landmarks? Perhaps information about error correction or overshoots should be included. Differences among individual travelers are again important. In particular, some people prefer "route" information that focuses on describing a sequential chain of landmarks and simple turn actions; others can understand and prefer some "survey" information about direct quantitative spatial relations among places in the environment.

\subsection{Wayfinding with Maps}

Cartographic maps are the quintessential example of information displays for wayfinding. There are a host of design issues for optimizing map effectiveness, such as legend and symbol design. The degree of schematic abstraction in map design is relevant. Maps used to wayfind need not 
communicate extremely detailed and precise metric information about distances and directions. Particularly when the map is used to navigate on a constrained path network, such as a subway system, most travelers may only want to know the connections among network segments - the quantitative distance between stops may be irrelevant, for instance. However, even though a subway map may not depict metric information accurately, it is a graphical display that necessarily depicts metric information, and some users of the map may interpret its metric relations inappropriately.

An important issue for maps used to wayfind is their orientation, in particular which direction on the map is placed at the top [10]. When maps are used outside the context of a particular surrounding environment, their orientation is primarily a mater of convention — orientations that differ from common convention (e.g. north at the top) do cause difficulties in such cases. But when a map is being used to actively guide navigational decisions in the surrounding environment, most people perform faster and more accurately when the top of the map is aligned with the forward direction of movement. In this case, the bottom is aligned with the backward direction, and left and right on the map are left and right in the surrounds. This is known as "forward-up" or "track-up" alignment. For most people, maps not so aligned must be physically or imaginally reoriented, that is if their misalignment is recognized (a minority of people are able to apply feature-matching strategies that obviate the need for realignment). These processes are cognitive costly and in fact lead to disorientation. The error and extra time caused by using misaligned maps is called an alignment effect. Figure 1 shows an example of misaligned youare-here (YAH) maps. 


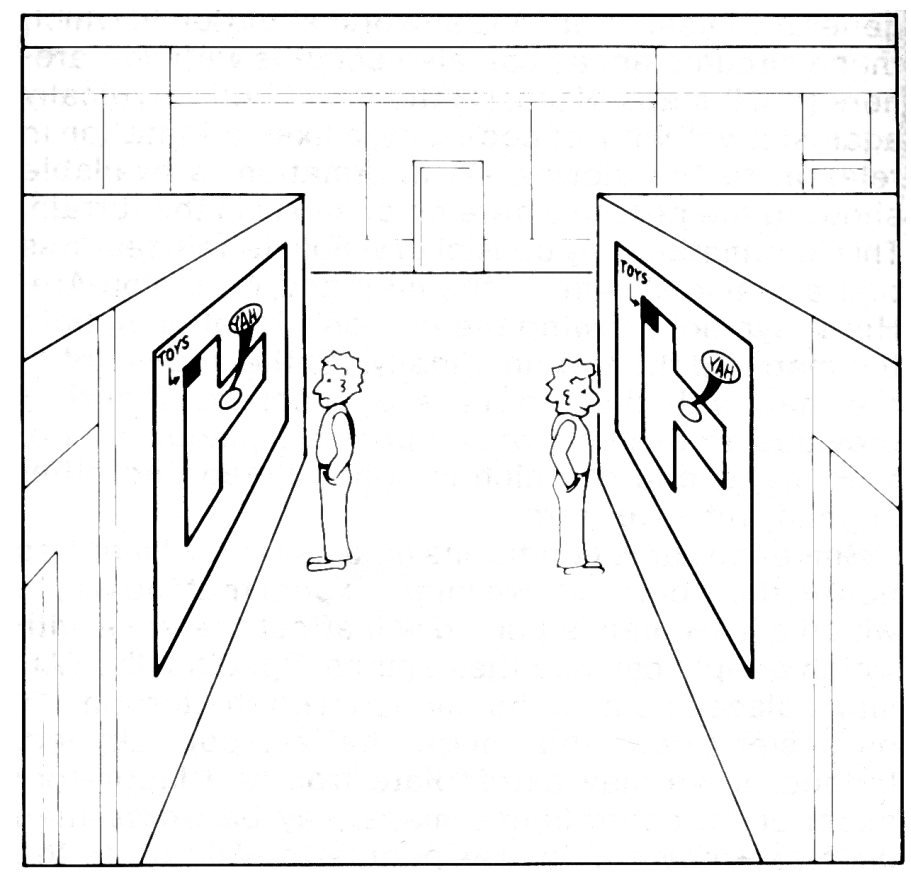

Figure 1. The orientation in which YAH maps are mounted leads to misaligned maps (from Levine, M. et al. Environment and Behavior, 16, 144, 1984. With permission).

Map alignment effects have implications for the design of digital navigation systems for automobiles and cell phones [11]. Even though track-up alignments are best for most people, research has shown that a significant minority of people prefers navigation maps such as these to be aligned in a fixed orientation, such as "north-up," probably because of the familiarity of looking at the map in a constant orientation. An interesting possibility is that a fixed alignment may better facilitate using maps to acquire knowledge of spatial layout- - to form cognitive maps. This suggests that optimal design for navigation systems should allow both variable and fixed orientations, controllable by the traveler.

3.3. Wayfinding Technologies for the Visually Impaired 
Providing for individuals with disabilities leads to some unique human factors issues. With respect to wayfinding, people with visual impairments (including blindness) face considerable difficulties wayfinding in cities, public buildings, and transit systems [12]. There is a fairly long history of research and development on the human factors of locomotion for those with visual impairments, including work on long canes, guide dogs, and auditory crossing signals. There is surprisingly little on the human factors of wayfinding for those with visual impairments, despite the powerful social, economic, and emotional importance of independent travel. Interestingly, tasks within the domain of locomotion for most of us are in a sense transformed into tasks within the domain of wayfinding for people with severe visual impairments. It is challenging to provide wayfinding information to those with visual impairments about opportunities in the environment and how to reach them. Clearly the information must be communicated in the auditory and tactile modalities. Technologies being developed and tested include so-called Personal Guidance Systems (PGS) that incorporate detailed spatial databases, GPSs, and inertial compasses, combined into a unit worn or carried by the traveler. Another example is Remote Infrared Auditory Signage (RIAS); the traveler carries a receiver that picks up infrared signals when pointed at transmitters posted around public environments (the presence of the transmitters is detected by a "sweeping" motion of the receiver). The transmitter sends information about the place where it is posted (such as the line number of a bus or the door of a public restroom); the information is communicated as speech to the traveler.

\section{Wayfinding in Virtual Environments}

Virtual environments (VEs) are computer-generated "worlds" that respond in real time to user behaviors. Users travel through "navigable VEs." Navigable VE systems come in a variety of 
types, including desktop, partially immersive, and fully immersive systems; differences among these types have important implications for wayfinding performance (and, of course, locomotion performance). Hardware and software characteristics of VEs limit the information they convey. Depending on their type, they provide limited visual detail, field-of-view, depth information, and sensorimotor channels (notably, with respect to orientation, limited kinesthetic and vestibular input). Even the most sophisticated fully immersive systems currently have such limitations.

Research has investigated how to increase the usability of navigable VEs, attempting to develop design guidelines for supporting orientation during wayfinding. Some research has investigated orientation in VEs in and of themselves; other research has investigated the effectiveness with which knowledge acquired in VE simulations transfers to the real world. Issues have included the fidelity or realism of the interface, the quality and amount of training in the system, the effects of various types of orientation cues, and the relevance of differences among individual users [13-15]. Wayfinding in VEs is improved when their design is informed by the same principles we discussed above that influence orientation in real environments, including differentiation, visual access, and layout complexity. Studies have indicated the importance of the presence, appearance, and location of landmarks in VEs, perhaps indicating they are even more important than in real environments. The availability of well-designed and placed maps, signs, and other semiotic wayfinding devices are important too. Furthermore, some research has investigated wayfinding aids and challenges unique to VEs, such as teleportation, rapid scale transformations, and flexibly modifiable maps and models that can be integrated within the virtual spaces they represent. Trails or footprints of user's trajectories could usefully guide search tasks in VEs. 
These various insights about how to ensure effective wayfinding in VEs have not yet been sufficiently exploited in their design and construction, however. Instead, attention has focused almost exclusively on technological aspects. Furthermore, much greater attention needs to be paid to ways that individuals differ with respect to wayfinding in VEs, which have been shown to account for large amounts of variance in performance in a given VE system or layout. These design shortcomings with respect to wayfinding are partly explained by the novelty of VE technologies and the challenges faced by their designers.

\section{Conclusions}

In this essay, we introduce the construct of navigation, and its components of wayfinding and locomotion. We summarize human factors associated with wayfinding, the goal-directed and planning component of navigation. We outline factors related to human psychology, such as orientation, attention, and the automaticity of wayfinding tasks; factors related to the environment, such as differentiation, visual access, layout complexity, and sign placement; and factors related to technology, such as the design of information displays in vehicle navigation systems. We specifically contrast verbal route directions and wayfinding with maps, and consider wayfinding technologies for those with visual impairments and for navigation in VEs.

While theoretical work strives to improve our understanding of wayfinding, tools and applications that have been developed attempt not only to support wayfinding behavior but also to train spatial skills (VEs are promising in the latter respect). Applications are limited mainly because they are insufficiently connected with the body of literature on theories of wayfinding and attend almost exclusively to its technological aspects. This may be partly explained by the novelty of these technologies and the technological challenges faced by their designers. It is 
clear, however, that the design of applications must be informed by basic research if it is to be optimized.

An important example is the issue of individual differences with respect to wayfinding behavior. This has been largely ignored in the development of applications. Several individual differences, such as gender, computer experience, and spatial skills have been identified as significantly impacting wayfinding performance. However, a large percentage of variation in performance is still unexplained. We conjecture that spatial behaviors expressed in particular detailed movement patterns could offer new insight into explaining individual differences.

Fortunately, market constraints are forcing greater attention because individual differences impact directly on the use of wayfinding support systems. Thus, in order to reach their potential, wayfinding applications require extensive usability studies. Even if traditional route following and map reading can largely be carried out without specific additional training, as part of our cultural inheritance, the use of some in-car navigation systems and VEs do require training in specific skills.

\section{References}

1. Montello, D.R., Navigation. In The Cambridge Handbook of Visuospatial Thinking, Miyake, A. and Shah, P., Eds., Cambridge University Press, Cambridge, in press.

2. Hill, K.A., Ed., Lost Person Behaviour, Ottawa, Ontario, The National Search and Rescue Secretariat, 1999.

3. Hegarty, M. et al., Development of a self-report measure of environmental spatial ability. Intelligence, 30, 425-447, 2002. 
4. Carpman, J.R. and Grant, M.A., Wayfinding: A broad view. In Handbook of Environmental Psychology, Bechtel, R.B. and Churchman, A., Eds., Wiley, New York, $2002,427-442$.

5. Weisman, J., Evaluating architectural legibility: Way-finding in the built environment. Environment and Behavior, 13, 189-204, 1981.

6. Arthur, P. and Passini, R., Wayfinding: People, Signs, and Architecture, McGraw-Hill Ryerson, Toronto, 1992.

7. Burnett, G. and Joyner, S., Route guidance systems: Getting it right from the driver's perspective. Journal of Navigation, 49, 169-177, 1996.

8. Streeter, L.A., Vitello, D. and Wonsiewicz, S.A., How to tell people where to go: comparing navigational aids. International Journal of Man/Machine Studies, 22, 549$562,1985$.

9. Allen, G.L., From knowledge to words to wayfinding: Issues in the production and comprehension of route directions. In Spatial Information Theory: A Theoretical Basis for GIS, Hirtle, S.C. and Frank, A.U., Eds., Springer-Verlag, Berlin, 1997, 363-372.

10. Levine, M., Marchon, I. and Hanley, G.L., The placement and misplacement of you-arehere maps. Environment and Behavior, 16, 139-157, 1984.

11. Aretz, A.J. and Wickens, C.D., The mental rotation of map displays. Human Performance, 5, 303-328, 1992.

12. Marston, J.R. and Church, R.L., A relative access measure to identify barriers to efficient transit use by persons with visual impairments. Journal of Disability and Rehabilitation, in press. 
13. Darken, R. and Peterson, B., Spatial orientation, wayfinding, and representation. In Handbook of Virtual Environments: Design, Implementation, and Applications, Stanney, K.M., Ed., Lawrence Erlbaum Associates, Mahwah, NJ, 2002, 493-518.

14. Ruddle, R.A., Payne, S.J. and Jones, D.M., Navigating buildings in 'desk-top' virtual environments: Experimental investigations using extended navigational experience. Journal of Experimental Psychology: Applied, 3, 143-159, 1997.

15. Waller, D., Hunt, E. and Knapp, D., The transfer of spatial knowledge in virtual environment training. Presence: Teleoperators and Virtual Environments, 7, 129-143, 1998. 